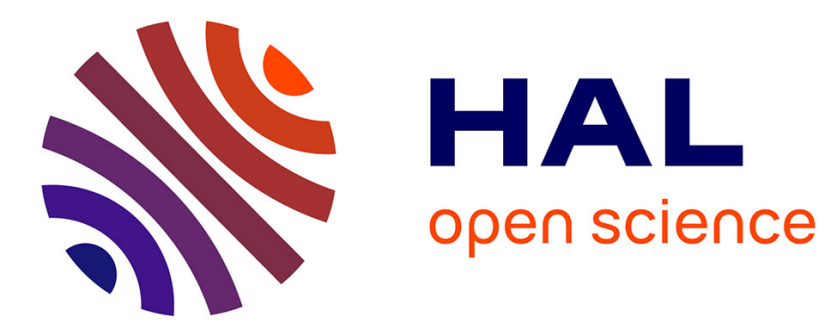

\title{
ELECTRON IMPACT EXCITATION OF 2pq AND 3pq IONS
}

C. Mendoza

\section{To cite this version:}

C. Mendoza. ELECTRON IMPACT EXCITATION OF 2pq AND 3pq IONS. Journal de Physique IV Proceedings, 1991, 01 (C1), pp.C1-61-C1-65. 10.1051/jp4:1991108 · jpa-00249746

\section{HAL Id: jpa-00249746 https://hal.science/jpa-00249746}

Submitted on 1 Jan 1991

HAL is a multi-disciplinary open access archive for the deposit and dissemination of scientific research documents, whether they are published or not. The documents may come from teaching and research institutions in France or abroad, or from public or private research centers.
L'archive ouverte pluridisciplinaire HAL, est destinée au dépôt et à la diffusion de documents scientifiques de niveau recherche, publiés ou non, émanant des établissements d'enseignement et de recherche français ou étrangers, des laboratoires publics ou privés. 
ELECTRON IMPACT EXCITATION OF $2 p^{q}$ AND $3 p^{q}$ IONS

\author{
C. MENDOZA \\ IBM Venezuela Scientific Center, po. Box 64778, Caracas 1060A, \\ Venezuela
}

\begin{abstract}
RESUME - Nous examinons le travail récent sur l'excitation par impact électronique des ions positifs de configurations $2 p^{q}$ et $3 p^{q}(q=1-5)$. Nous évaluons les articles publiés depuis la denière révision menée pour l'Atomic Data W'orkshop tenu à Oxford en aoüt 1987.

ABSTRACT - Recent work on electron impact excitation of positive ions with $2 p^{q}$ and $3 p^{q}$ configurations $(q=1-5)$ is reviewed. We assess papers published since the last revision carried out for the Atomic Data Workshop held at Oxford in August 1987.
\end{abstract}

\title{
1 - INTRODUCTION
}

The present report updates two earlier revisions on electron impact excitation of positive ions with $2 p^{q}$ and $3 p^{q}$ electronic configurations. They were presented at the Atomic Data Workshops held at the Daresbury Laboratory in March 1985/1/ and at St Catharine College, Oxford, in August 1987/2/. Even though accurate cross sections for transitions in such ions are required in low and high temperature astrophysical and laboratory plasma diagnostics, in the past two years there has been a considerable decrease in reported work. This is perhaps due to lack of interest since these ions have received a lot of attention in the past, and the present trend is to attempt more ambitious calculations for larger systems. However, an exha'ustive examination carried out by Burke et al $/ 17 /$ on the vell studied O III system has shown that a very elaborate calculation must be performed to pin down the sensitive transitions. Furthermore, new extensive experimental work on the electron impact excitation of O I (see section 2.1) has resulted in cross sections which do not always agree with theoretical results.

In our opinion a lot more work is necessary before we can be confident on the data quality we assert amongst our customers. In this respect, an innovative treatment of the excitation of very highly ionised ions using a relativistic distorted wave approximation $/ 37,38 /$ has shown that even for such systems resonances can play a key role in the excitation process of forbidden transitions. These results call for a revision of many calculations where resonances have generally been assumed to have a negligible contribution.

We will structure the following discussion in three areas: neutrals and lowly ionised species; highly ionised systems and very highly ionised systems.

\section{2 - NEUTRALS AND LOWLY IONISED SPECIES}

\section{$2.10 I$}

Encouraged by the prominence of O I emission lines in terrestial airglow and polar aurora, extensive measurements have been performed on the electrom impact excitation of allowed and forbidden transitions of this system. Five papers were reported in our earlier revision $/ 2 /$ and seven related experimental results have been published since then. We discuss them briefly.

Due to difficulties in analysing the observed intensities for the ${ }^{3} P \rightarrow 3 s^{3} S^{0}(1304 \AA)$ and ${ }^{3} P \rightarrow 3 d^{3} D^{0}(989$ $\AA$ ) transitions using published cross sections (see references in $/ 2 /$ ), Vaughan and Doering $/ 3 /$ have reported new measurements in the electron energy range $20 \mathrm{eV}-200 \mathrm{eV}$ using the method of cross-beams electron energy-loss spectrometry. They have carefully reanalysed previous data for the ${ }^{3} P \rightarrow{ }^{3} S^{0}$ differential cross section $(d c s)$ using computer fits in order to employ this cross section as a standard. This procedure only led to small changes $(<15 \%)$. On the other hand, the integral cross section (ics) obtained for the ${ }^{3} P \rightarrow{ }^{3} D^{0}$ is found to be a factor of 3 lower that previous experimental data and it leads to a considerable reduction of the discrepancy with the airglow data.

Vaughan and Goering/4/ obtained $d c s$ and ics for transitions from the ground state to the $3 s^{\prime \prime}{ }^{3} P^{0}$, $2 s 2 p^{53} P^{0}$ and $4 d^{\prime 3} P^{0}$ autoionising states and the first five members of the $n d^{3} D^{0}$ series. A range of 30 to 200 
Table 1. Ratio of the effective collision strength at $T=10000 \mathrm{~K}$ for transitions within the $3 p^{3}$ ground configuration of ions of the $P$ sequence calculated in a distorted wave approximation / 39 / to that recently calculated using the close-coupling method $/ 17,18,19 /$. Large differences are observed for most transitions.

\begin{tabular}{llll}
\hline Transition & Cl III & Ar IV & K V \\
\hline${ }^{4} S_{3 / 2}^{0}-{ }^{2} D_{3 / 2}^{0}$ & 0.93 & 0.44 & 0.53 \\
${ }^{4} S_{3 / 2}^{0}-{ }^{2} D_{5 / 2}^{0}$ & 0.92 & 0.44 & 0.53 \\
${ }^{2} D_{3 / 2}^{0}-{ }^{2} D_{5 / 2}^{0}$ & 0.71 & 0.22 & 0.18 \\
${ }^{4} S_{3 / 2}^{0}-{ }^{2} P_{1 / 2}^{0}$ & 1.50 & 1.45 & 0.47 \\
${ }^{4} S_{3 / 2}^{0}-{ }^{2} P_{3 / 2}^{0}$ & 1.51 & 1.44 & 0.47 \\
${ }^{2} D_{3 / 2}^{0}-{ }^{2} P_{1 / 2}^{0}$ & 0.75 & 0.60 & 0.78 \\
${ }^{2} D_{3 / 2}^{0}-{ }^{2} P_{3 / 2}^{0}$ & 0.87 & 0.50 & 0.55 \\
${ }^{2} D_{5 / 2}^{0}-{ }^{2} P_{1 / 2}^{0}$ & 0.88 & 0.58 & 0.52 \\
${ }^{2} D_{5 / 2}^{0}-{ }^{2} P_{3 / 2}^{0}$ & 0.79 & 0.56 & 0.69 \\
${ }^{2} P_{1 / 2}^{0}-{ }^{2} P_{3 / 2}^{0}$ & 0.76 & 0.26 & 0.45 \\
\hline
\end{tabular}

eV for the incident electron energy was considered. The transition to the $3 s^{\prime \prime}{ }^{3} P^{0}$ state $(878 \AA)$ was found to be a factor of 3 smaller than previous results.

Gulcicek and Doering /5/ revised the cross sections for the allowed ${ }^{3} P \rightarrow{ }^{3} S^{0}$ and ${ }^{3} P \rightarrow{ }^{3} D^{0}$ transitions at low energies $(\leq 20 \mathrm{eV})$ using a new experimental set up. The apparatus has been designed to measure forbidden transitions at low energies. Whilst the new cross sections agree at $30 \mathrm{eV}$ and above with the results by Vaughan and Doering $/ 3 /$, a.t the lower energies they are found to be a factor of 2 or 3 higher. The accuracy of the cross section for the transition ${ }^{3} P \rightarrow{ }^{3} S^{0}$ is now quoted to be better than $30 \%$.

Absolute $d c s$ and ics have been measured for the following forbidden transitions: ${ }^{3} P \rightarrow{ }^{3} P$ and ${ }^{3} P \rightarrow{ }^{5} P$ in the range 13.87-100 eV $/ 6 / ;{ }^{3} P \rightarrow{ }^{1} D$ and ${ }^{3} P \rightarrow{ }^{1} S$ in the range $4.0-30 \mathrm{eV}$ with an accuracy of around $40 \%$ $/ 7 /$ and the ${ }^{3} P \rightarrow{ }^{5} S^{0}$ in the range $13.9-30 \mathrm{eV}$ also quoted at $40 \% / 8 /$. The ${ }^{3} P \rightarrow{ }^{5} P$ emission cross section measured by Germany et al /9/ cannot be compared with the excitation data due to cascade contributions.

The challenge to reproduce theoretically these new and extensive measurements has been taken up by Tayal and Henry $/ 10,11 /$. Cross sections for the ${ }^{3} P \rightarrow{ }^{3} S^{0}$ and ${ }^{3} P \rightarrow{ }^{3} P$ cross sections have been calculated in $3 \mathrm{CC}+\mathrm{CI}, 7 \mathrm{CC}+\mathrm{CI}$ and $12 \mathrm{CC}+\mathrm{CI}$ approximations. Good agreement is obtained for the former transition but for the latter the theoretical results are a factor of 2 to 3 higher at energies $E>20 \mathrm{eV}$. The $12 \mathrm{CC}+\mathrm{CI}$ approximation has been used to calculate cross sections for transitions from the ground state to the $3 s^{5} S^{0}, 3 p{ }^{5} P, 4 s^{3} S^{0}$, $4 p^{3} P, 3 d^{3} D^{0}$ and $3 s^{\prime}{ }^{3} D^{0}$ states. Agreement with experiment is varied.

\subsection{II}

The recent measurement of the absolute cross section for the $2 s^{2} 2 p^{2} P^{0} \rightarrow 2 s 2 p^{2}{ }^{2} D$ transition in C II /12/ is the first reported experimental result for a boron-like ion. They have used the colliding-beams method whereby the cross section is determined by monitoring the $134 \mathrm{~nm}$ photons arising from the decay of the excited ion. An important problem in the experiment is to determine the population of the ${ }^{4} P$ metastable state which is determined from a theoretical estimate /13/ of its lifetime. Good agreement is found with a close-coupling result $/ 14 /$, but a comparison with the more recent $8 \mathrm{CC}+\mathrm{CI}$ results by Hayes and Nussbaumer /15/ is not carried out since the latter authors only published effective collision strengths.

Luo and Pradhan /16/ have recently performed the largest (10CC+CI) calculation on this ion, and collision strengths are given for the fine-structure transitions arising from the 10 target terms. Good agreement is found for the ${ }^{2} P_{1 / 2}^{0} \rightarrow{ }^{2} P_{3 / 2}^{0}$ transition within the ground state with previous $8 \mathrm{CC}+\mathrm{CI}$ results $/ 15 /$. Effective collision strengths are not given.

\section{$2.3 N$ III and $O I V$}

Luo and Pradhan /16/ also treated N III and O IV in 8CC+CI approximations. They discuss to some extent the problem of bound-state resonances which can introduce unexpected errors in the temperature behaviour of effective collision strengths. 
Table 2. Comparison of total collision strengths for the $2 s^{2} 2 p^{2}-2 s 2 p^{3}$ optically allowed transitions in $\mathrm{Mg}$ VII as given by Burgess et al /25/. Results are calculated by different methods: distorted wave results /26) (DW); 9CC using the IMPACT code (IMP); 9CC R-matrix results using the standard asymptotic package by Crees / 40/ (RN1) and R-matrix results by Aggarwal /27/ using a Coulomb-functions asymptotic code (RM2). Whilst there is good agreement among DW, IMIP and RM1, noticeable differences are found with RM2; these discrepancies are believed to be due to the asymptotic package used in $/ 27 /$.

\begin{tabular}{lllll}
\hline Transition & DW & INIP & RMI & RM2 \\
\hline${ }^{3} P-{ }^{3} D^{0}$ & 3.38 & 3.29 & 3.27 & 2.86 \\
$3^{3} P-{ }^{3} P^{0}$ & 3.73 & 3.65 & 3.62 & 3.03 \\
${ }^{3} P-{ }^{3} S^{0}$ & 2.92 & 2.95 & 2.91 & 2.65 \\
$1 D-{ }^{1} D^{0}$ & 3.48 & 3.50 & 3.45 & 3.00 \\
$1 D-1 P^{0}$ & 2.05 & 2.04 & 2.01 & 1.80 \\
$1 S-{ }^{1} P^{0}$ & 0.68 & 0.68 & 0.68 & 0.60 \\
\hline
\end{tabular}

\section{$2.4 O I I I$}

Burke et al /17/ have carried out an exhaustive study of the transitions involving the ${ }^{3} P,{ }^{1} D,{ }^{1} S$ and ${ }^{5} S^{0}$ states of O III. For this purpose they have considered 12 target states in different CI representations; they present comparisons of collision strengths with those from previous close-coupling calculations and of bound states of 0 II with spectroscopic data. The recommended effective collision strengths are probably the most reliable set of results for any $n p^{q}$ ion.

\subsection{P-like ions}

The transitions within the $3 p^{3}$ configuration of $\mathrm{Cl}$ III, Ar IV and $\mathrm{K} \mathrm{V}$ have been recently considered /18, 19 , $20 \%$ As seen in Table 1 large differences are found with effective collision strengths estimated from previous DW data $/ 39 /$. Since these ions are difficult to treat properly the reported effective collision strengths cannot be reliably ranked.

\section{3 - HIGHLY IONISED IONS}

\subsection{Fluorine-like ions}

Effective collision strengths have been recently calculated for transitions from the ground state to excited LS states of the following F-like ions: Mg IV (11CC+CI) /21/; Si VI (16CC+CI) /22/; S VIII (12CC+CI)/23/ and Ti XIV (12CC+CI) /24/. A suggested continuation of this work would be to compute rates for transitions arising from excited states and to provide the fine-structure components.

\section{$3.2 \mathrm{Mg} H$}

Burgess et al $/ 2 \tilde{\Xi} /$ have carried out a critical assessment of the collisional work for transitions between the $2 s^{2} 2 p^{2}$ and $2 s 2 p^{3}$ configurations of $\mathrm{Mg} V \mathrm{VIT}$. This has been motivated by unexpected large differences $(\sim 35 \%)$ for the allowed transitions between the DW data of Mason and Bhatia /26/ and the close-coupling (R matrix) results of Aggarwal $/ 27 /$. By performing detailed comparative calculations using different numerical approximations (see Table 2), they present conclusive evidence to show that the differences are mostly due to a unreliable method used by Aggarwal in the asymptotic region. Since this approach has been used in other R-matrix calculations (e.g., Ne V, Si II), they warn against possible inaccurate collision strengths for allowed transitions.

\section{3. $\mathrm{Fe} X I I$}

Tayal et al /28/ have calculated effective collision strengths for the fine-structure structure transitions of the $3 p^{3}$ ground configuration of $\mathrm{Fe} \mathrm{XII}$ in a $7 \mathrm{CC}+\mathrm{CI}$ approximation. Relativistic effects in the target are included 16rough a Breit-Pauli formulation. This approach has been subsequently extended to transitions to the $3 s 3 p^{4}$ excited configuration /29/. Large differences are found with the DW results of Flower /30/, particularly for the level populations of the ground configuration. 


\section{$3.4 \mathrm{Fe}$ XIII}

Fawcett and Mason /31/ have introduced the technique of Slater parameter optimisation into a DW calculation to obtain collision strengths for the fine-structure transitions from the ground configuration $3 s^{2} 3 p^{2}$ to the excited $3 s 3 p^{3}$ and $3 s^{2} 3 p 3 p$ configurations of Fe XIII. Slater parameter optimisation is a well established technique in atomic structure calculations, and it should prove useful in collision calculations of complex ions where even elaborate $a b$ initio approaches come short of providing realistic representations for the contributing interactions. However, a comparison with previous work /32, 33/ was not presented which would have been useful in appreciating the magnitude of the differences brought about by this new method.

\section{4 - VERY HIGHLY IONISED IONS}

\subsection{C-like ions}

In a similar approach to that used for ions of the boron sequence /34/, Bhatia et al /35/ have computed collision strengths for transitions in Ar XIII, Ti XVII, Fe XXI, Zn XXV, Se XXIX and Kr XXXI, involving the 46 finestructure levels belonging to the following configurations: $2 s^{2} 2 p^{2}, 2 s 2 p^{3}, 2 p^{4}, 2 s^{2} 2 p 3 s, 2 s^{2} 2 p 3 p$ and $2 s^{2} 2 p 3 d$. A DW approximation is used with relativistic corrections in the target. We expect these results to be accurate to within the level of accuracy expected for the DW method (see comments in /2/). A similar computation for $\mathrm{N}$-like ions is to be published.

\section{$4.2 \mathrm{Fe} X I X$}

The method described in 4.1 is employed to compute collision strengths for fine-structure transitions between the $2 p^{4} \rightarrow 2 p^{3} 3 d$ configurations of Fe XIX $/ 36 /$.

\subsection{Se XXVII}

A very interesting new technique has been used by Reed et al /37/ to calculate excitation rates for the $n=2$ transitions of very highly ionised systems such as O-like Se. They are particularly interested in the contribution of resonances. The nonresonant cross sections are calculated in a relativistic DW approximation with CI target wavefunctions obtained from a Dirac-Fock atomic structure code. Resonances are calculated in the isolated-resonance approximation neglecting interference with the non-resonant part, and radiative decay of the autoionising state to singly excited states is also taken into account. Large contributions from resonances are found for the dipole forbidden transitions; in particular, for the highly forbidden $J=0 \rightarrow J^{\prime}=0$ the resonance enhancement is as large as $\sim 10^{4}$.

\subsection{F-like ions}

The method described in section 4.3 is extended to transitions within $n=2$ of F-like ions, namely Fe, Se, Mo, $\mathrm{Ag}$, Xe and $\mathrm{Eu} / 38 /$. Again, large increases caused by resonances are found for forbidden transitions.

\section{ACKNOWLEDGEMENTS}

Part of the work for this paper was carried out during a visit to the Observatoire de Paris, Meudon, in July 1989. Financial support from the Observatoire is gratefully acknowledged.

\section{REFERENCES}

11/ Mendoza, C., in Atomic Data Workshop: Low energy collision theory techniques for atomic excitation and radiative data, Eissner, W.B. (ed), DL/SCI/R24 (Daresbury Laboratory) (1986) 80.

/2/ Mendoza, C., in Atomic Data Workshop: Assessment of data for photo-ionisation and photo-excitation and for electron impact excitation of atomic ions, Eissner, W. and Kingston A.E. (eds.), Daresbury Laboratory (1988) 136.

/3/ Vaughan, S.O. and Doering, J.P., J. Geophys. Res. 92 (1987) 7749.

(4) Vaughan, S.O. and Doering, J.P., J. Geophys. Res. 93 (1988 289.

15/ Gulcicek, E.E. and Doering, J.P., J. Geophys. Res. 93 (1988) 5879.

/6/ Gulcicek, E.E., Doering, J.P. and Vaughan, S.O., J. Geophys. Res. 93 (1988) 5885.

17/ Doering, J.P. and Gulcicek, E.E., J. Geophys. Res. 94 (1989) 1541.

/8/ Doering, J.P. and Gulcicek, E.E., J. Geophys. Res. 94 (1989) 2733. 
19/ Germany, G.A., Anderson, R.J. and Salamo, G.J., J. Chem. Phys. 89 (1988) 1999.

10/ Tayal, S.S. and Henry, R.J.W., Phys. Rev. A 38 (1988) 5945.

111/ Tayal, S.S. and Henry, R.J.W., Phys. Rev. A 39 (1989) 4531.

/12/ Lafyatis, G.P. and Kohl, J.L., Phys. Rev. A 36 (1987) 59.

/13/ Nussbaumer, H. and Storey P.J., Astron. Astrophys. 71 (1979) L5.

114/ Magee, N.H., Mann, J.B., Merts, A.L. and Robb, W.D., Los Alamos Informal Report, LA-6691-MS (1977).

15/ Hayes, M.A. and Nussbaumer, H., Astron. Astrophys. 134 (1984) 193.

16/ Luo, D. and Pradhan, A.K., Phys. Rev. A (1990) in press.

117/ Burke, V.M., Lennon, D.J. and Seaton; M.J., MNRAS 236 (1989) 353.

/18/ Butler, K. and Zeippen, C.J., Astron. Astrophys. 208 (1989) 337.

/19/ Zeippen, C.J., Butler, K. and Le Bourlot J., Astron. Astrophys 188 (1987) 251.

120/ Butler, K., Zeippen, C.J. and Le Bourlot, J., Astron. Astrophys. 203 (1988) 189.

/21/ Mohan, M., Baluja, K.L. and Hibbert, A., Physica Scripta 38 (1988) 699.

/22/ Mohan, M. and Le Doumeuf M, Astron. Astrophys. 227 (1989) 285.

/23/ Mohan, M., Baluja, K.L. and Hibbert, A., J. Phys. B 20 (1987) 2565.

/24/ Mohan, M., Baluja, K.L. and Hibbert, A., Physica Scripta 40 (1989) 53.

/25/ Burgess, A., Mason, H.E. and Tully, J.A., to be published.

126/ Mason, H.E. and Bhatia, A.K., MNRAS 184 (1978) 423.

127/ Aggarwal, K.M., Astron. Astrophys. 146 (1985) 149.

/28/ Tayal, S.S., Henry, R.J.W. and Pradhan, A.K., Astrophys. J 319 (1987) 951.

/29/ Tayal, S.S. and Henry, R.J.W., Astrophys. J 329 (1988) 1023.

/30/ Flower, D.R., Astron. Astrophys. 54 (1977) 163.

/31/ Fawcett, B.C. and Mason, H.E., Atomic Data Nuclear Data Tables (1990) in press.

132/ Flower, D.R. and Pineau Des Forets, G., Astron. Astrophys. 24 (1973) 181.

/33/ Flower, D.R. and Nussbaumer, H., Astron. Astrophys. 31 (1974) 353.

134/ Bhatia, A.K., Feldman, U. and Seely, J.F., Atomic Data Nuclear Data Tables 35 (1986) 319.

/35/ Bhatia, A.K., Seely, J.F. and Feldman, U., Atomic Data Nuclear Data Tables 36 (1987) 453.

/36/ Bhatia, A.K., Fawcett, B.C., Lemen, J.R., Mason, H.E. and Phillips, K.J.H., MNR \&S 240 (1989) 421.

137/ Reed, K.J., Chen, M.H. and Hazi, A.U., Phys. Rev. A 38 (1988) 3319.

/38/ Reed, K.J., Chen, M.H. and Hazi, A.U., Phys. Rev. A 36 (1987) 3117.

/39/ Krueger, T.K. and Czyzak, S.J., Proc. Roy. Soc. Lond. A 318 (1970) 531.

/40/ Crees, M.A., Comput. Phys. Commun. 19 (1980) 103. 